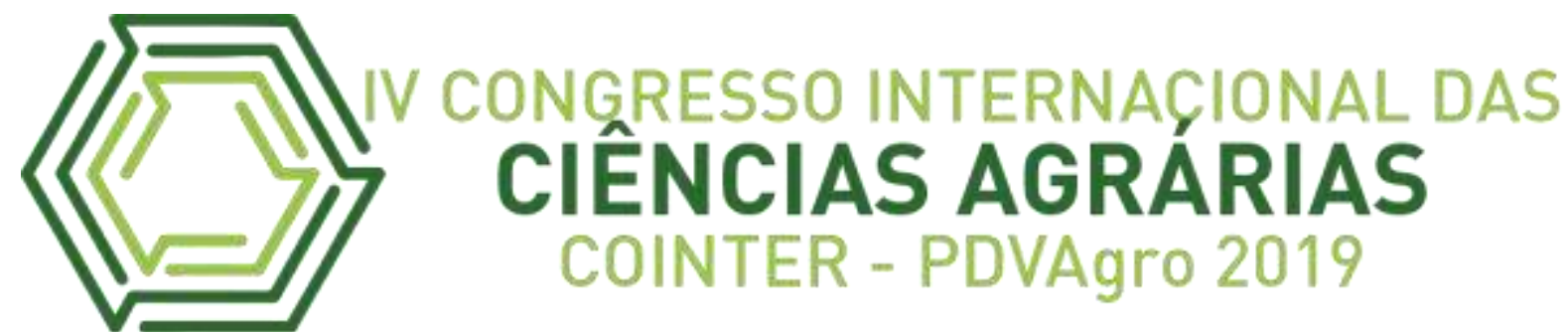

\title{
FITOSSOCIOLOGIA DA POPULAÇÃO DE Manilkara huberi (Ducke) A. Chev. EM ÁREA DE MANEJO FLORESTAL NA FLONA DO TAPAJÓS
}

\section{FISOCIOLOGÍA DE LA POBLACIÓN DE Manilkara huberi (Ducke) A. Chev. EN ÁREA DE MANEJO FORESTAL EN TAPAJÓS FLONA}

\section{PHYSOSOCIOLOGY OF THE POPULATION OF Manilkara huberi (Ducke) A. Chev. IN FOREST MANAGEMENT AREA IN TAPAJÓS FLONA}

\author{
Osvaldo Júnior Moraes Moreira ${ }^{1}$; Ana Kaira Canté da Conceição²; Hanna Kassia \\ Machado da Silva ${ }^{3}$; Jose Humberto Fernandes da Rocha Sobrinho ${ }^{4}$; Nilton Junior Lopes \\ Rascon $^{5}$
}

DOI: https://doi.org/10.31692/2526-7701.IVCOINTERPDVAgro.2019.0160

\begin{abstract}
Resumo
O objetivo do presente estudo foi avaliar a fitossociologia da espécie Manilkara huberi (Ducke) A. Chev., em uma área de manejo florestal da Flona do Tapajós em Belterra - Pará. A referida espécie é uma das mais exploradas pela Cooperativa Mista da Flona do Tapajós, que é a responsável por realizar nessa área o manejo florestal comunitário, em escala empresarial, adotando-se a técnica exploratória de impacto reduzido (EIR). A maçaranduba, como é tradicionalmente conhecida é uma Sapotaceae altamente valorizada tanto no mercado nacional, quanto no internacional, devido principalmente às suas características de madeira pesada, dura e resistente, com densidade básica de $0,87 \mathrm{~g} . \mathrm{cm}^{3}$, indicada para uso na construção civil, confecção de dormentes, estacas, pontes, cercas e moirões. Dessa forma, compreende-se a relevância de estudar o seu comportamento, para que seja possível a aplicação de manejo adequado para a espécie de forma a garantir a sua conservação na estrutura florestal. Os dados deste trabalho foram obtidos na UPA 12 da Unidade de Manejo Florestal do Anambé, que possui área total de 1.723,66 ha, subdivida em 15 UT's irregulares de áreas variadas entre 74,33 ha e 177,61 ha, onde foi realizado o inventário 100\%, considerando indivíduos com DAP $\geq 35 \mathrm{~cm}$. Na análise dos dados, calculou-se a abundância, dominância, volume e distribuição diamétrica da espécie. A densidade encontrada para a maçaranduba foi de 1,73 árvores/ha e volume de 10,46 $\mathrm{m}^{3} / \mathrm{ha}$, sendo esta a espécie mais abundante na área de estudo, com 2.295 indivíduos. A $M$. huberi esteve presente em todas as classes diamétricas consideradas, tendo maior concentração nos intervalos de $55-75 \mathrm{~cm}$ de diâmetro e apresentando distribuição espacial de forma agrupada. A espécie apresentou alta
\end{abstract}

\footnotetext{
1 Engenharia Florestal, Universidade Federal do Oeste do Pará, jumoreira3133@gmail.com Engenharia Florestal, Universidade Federal do Oeste do Pará, anakaira17@gmail.com Engenharia Florestal, Universidade Federal do Oeste do Pará, rhanna_ptr@hotmail.com Agronomia, Universidade Federal do Oeste do Pará, humbertofrs18@gmail.com

Analista Ambiental, Instituto Chico Mendes de Conservação da Biodiversidade, nilton.rascon@icmbio.gov.br
} 
abundância na área, bem como grande estoque de volumetria aliada à tendência ao J-invertido na distribuição diamétrica, o que sugere a existência de um balanço entre o recrutamento e a mortalidade da espécie, padrão usual das florestas tropicais inequiâneas.

Palavras-Chave: Fitossociologia, maçaranduba, manejo florestal, Flona do Tapajós.

\section{Resumen}

El objetivo del presente estudio fue evaluar la fitosociología de la especie Manilkara huberi (Ducke) A. Chev., En un área de manejo forestal de Flona do Tapajós en Belterra - Pará. Tapajós, que es responsable de realizar el manejo forestal comunitario en esta área a escala corporativa, utilizando la técnica exploratoria de impacto reducido (EIR). Maçaranduba, como se le conoce tradicionalmente, es una Sapotaceae muy valorada en los mercados nacionales e internacionales, principalmente debido a sus características de madera pesada, dura y resistente, con una densidad básica de $0,87 \mathrm{~g} . \mathrm{cm}^{3}$, indicada para su uso en la construcción. construcción, fabricación de durmientes, estacas, puentes, cercas y piquetes. Por lo tanto, se entiende la relevancia de estudiar su comportamiento, de modo que sea posible aplicar un manejo apropiado a la especie para asegurar su conservación en la estructura forestal. Los datos de este trabajo se obtuvieron de UPA 12 de la Unidad de Manejo Forestal de Anambé, que tiene un área total de 1,723.66 ha, subdividida en 15 TUU irregulares que van desde 74.33 ha a 177.61 ha, donde estaba Se realizó un inventario del 100\%, considerando individuos con DAP $\geq 35 \mathrm{~cm}$. En el análisis de datos, se calculó la distribución de abundancia, dominancia, volumen y diámetro de las especies. La densidad encontrada para maçaranduba fue de 1.73 árboles / ha y un volumen de $10.46 \mathrm{~m}^{3}$ / ha, que es la especie más abundante en el área de estudio, con 2,295 individuos. M. huberi estuvo presente en todas las clases diametrales consideradas, presentando una mayor concentración en los intervalos de 55 a 75 $\mathrm{cm}$ de diámetro y presentando una distribución espacial agrupada. La especie mostró una gran abundancia en el área, así como un gran stock volumétrico combinado con la tendencia $\mathbf{J}$ invertida en la distribución del diámetro, lo que sugiere la existencia de un equilibrio entre el reclutamiento y la mortalidad de la especie, el patrón habitual de los bosques tropicales desiguales.

Palabras Clave: Fitosociología, maçaranduba, manejo forestal, Flona do Tapajós.

\section{Abstract}

The objective of the present study was to evaluate the phytosociology of the species Manilkara huberi (Ducke) A. Chev. in a Flona do Tapajós forest management area in Belterra - Pará. This species is one of the most explored by the Flona do Mista Mixed Cooperative. Tapajós, which is responsible for conducting community-based forest management in this area on a corporate scale, using the reduced impact exploratory technique (EIR). Maçaranduba, as it is traditionally known, is a highly valued Sapotaceae in the national and international markets, mainly due to its characteristics of heavy, hard and resistant wood, with a basic density of $0.87 \mathrm{~g} . \mathrm{cm}^{3}$, indicated for use in construction, making sleepers, stakes, bridges, fences and pickets. Thus, it is understood the relevance of studying its behavior, so that it is possible to apply appropriate management for the species in order to ensure its conservation in the forest structure. The data from this work were obtained from UPA 12 of the Anambé Forest Management Unit, which has a total area of 1,723.66 ha, subdivided into 
15 irregular TUUs ranging from 74.33 ha to 177.61 ha, where it was $100 \%$ inventory was performed, considering individuals with $\mathrm{DBH} \geq 35 \mathrm{~cm}$. In the data analysis, the abundance, dominance, volume and diameter distribution of the species were calculated. The density found for maçaranduba was 1.73 trees / ha and volume of $10.46 \mathrm{~m}^{3} /$ ha, which is the most abundant species in the study area, with 2.295 individuals. M. huberi was present in all diametric classes considered, having higher concentration in the $55-75 \mathrm{~cm}$ diameter intervals and presenting spatial distribution in a grouped way. The species showed high abundance in the area, as well as large volumetric stock combined with the inverted J-tendency in the diameter distribution, which suggests the existence of a balance between the recruitment and mortality of the species, the usual pattern of unequal tropical forests.

Keywords: Phytosociology, maçaranduba, forest management, Flona do Tapajós.

\section{Introdução}

Considerada o maior estoque natural de diversidade vegetal do mundo, a Floresta Amazônica apresenta uma rica composição florística nas suas mais variadas tipologias florestais, além de elevado grau de endemismo (VIEIRA et al., 2013).

De acordo com os autores, as espécies florestais de maior predominância na Amazônia representam para a região uma importante fonte de recursos naturais para as populações tradicionais e desenvolvimento local, tanto através dos produtos florestais madeireiros quanto dos não madeireiros. No entanto, Silva et al. (2015) destacam que apesar da complexidade das interações entre as populações bióticas e abióticas, o equilíbrio ecológico oriundo deste processo é considerado frágil.

No Pará, o setor florestal é uma das atividades econômicas mais importantes devido à grande geração de emprego, renda e divisas, além de ser a responsável por conceder ao estado paraense o título de maior produtor e exportador de madeira tropical do país. (Santana et al. 2012).

Entretanto, para que nessa região haja um aproveitamento racional, conservação da biodiversidade e produção contínua de madeira, faz-se necessária a prática do manejo florestal sustentável, visto que este permite o fornecimento de benefícios tão logo haja um mínimo planejamento para o aproveitamento de seus recursos (LIMA et al., 2018).

Ao compreender tal proposta, a Cooperativa Mista da Flona do Tapajós (COOMFLONA), instalada no município de Belterra - PA, realiza desde o ano de 2005 o manejo florestal comunitário em caráter empresarial com a adoção da exploração de impacto reduzido (EIR) na Floresta Nacional do Tapajós (SANTOS et al., 2018). 
Dentre as inúmeras espécies exploradas na mesma, destaca-se a Manilkara huberi (Ducke) A. Chev., tradicionalmente conhecida como maçaranduba, que segundo Cruz (2016) é uma das espécies mais exploradas em toda a Amazônia e comercializada tanto no mercado nacional, quanto no internacional, sendo apresentado pelo autor o valor de $3.614 .811 \mathrm{~m}^{3} \mathrm{de}$ madeira em tora da espécie exploradas de forma legal no período de 2006 a 2015 (RIBEIRO et al., 2013).

Considerando, portanto, a importância da espécie para a economia paraense, aliada à técnica de EIR utilizada pela COOMFLONA, destaca-se a importância de estudos que sirvam como base para o conhecimento sobre a estrutura da floresta e sua relação com a diversidade e produtividade da mesma (RIBEIRO et al., 2013).

Com base neste contexto e buscando contribuir para o conhecimento sobre o manejo de espécies nativas da Amazônia pouco estudadas e em concordância com o plano de ação para o desenvolvimento sustentável - Agenda 2030 - a qual é detentora de 17 objetivos de desenvolvimento sustentável (ODS), considera-se neste trabalho o objetivo 15 da mesma, onde trata-se da proteção, recuperação e promoção do uso sustentável dos ecossistemas terrestres, gerenciamento das florestas de forma sustentável, combate a desertificação e reversão da degradação da terra e detenção da perda de diversidade, objetivou-se com o presente estudo avaliar a fitossociologia da população de $M$. huberi em uma área de manejo florestal da Flona do Tapajós em Belterra - Pará.

\section{Fundamentação Teórica}

De acordo com Castro \& Carvalho (2014) o conhecimento sobre a estrutura de florestas tropicais é de fundamental importância para se adotar um sistema de manejo com base no rendimento sustentável. A análise estrutural é a base para os critérios de colheita do manejo florestal, permitindo estimar o estágio de desenvolvimento da floresta, além de auxiliar na aplicação de métodos silviculturais que melhorem a qualidade e produtividade da mesma.

A estrutura de uma população também é considerada determinante para compreender os processos que regulam uma comunidade e para a conservação de espécies (Vieira et al., 2013). Entendido isto, Castro \& Carvalho (2014) afirmam que as espécies que mais necessitam ser estudadas estruturalmente são aquelas mais colhidas e comercializadas, pois são as que mais têm suas populações afetadas, como no caso da maçaranduba. 
A referida espécie pertence à família Sapotaceae e seus indivíduos podem atingir até $50 \mathrm{~m}$ de altura e $300 \mathrm{~cm}$ de diâmetro (CRUZ, 2016). A M. huberi é bastante valorizada no mercado, devido principalmente às suas características de madeira pesada, dura e resistente, com densidade básica de 0,87 g.cm³ , tendo como principais finalidades a construção civil, confecção de dormentes, estacas, pontes, cercas e moirões (CASTRO \& CARVALHO, 2014). A maçaranduba é caracterizada por possuir fuste ereto e cilíndrico, com ritidoma marrom acinzentado a escuro ou avermelhado, com fissuras profundas (AZEVEDO et al., 2008). Segundo os mesmos autores, esta espécie ocorre na Amazônia, especialmente em florestas de terra firme, no entanto, pode ser encontrada na América Central, América do Sul e Caribe. Os autores afirmam ainda, que a maçaranduba é considerada uma espécie clímax no grupo sucessional. Além disto, é hermafrodita e algumas de suas populações florescem anualmente, enquanto outras florescem a cada cinco anos.

Considerando todas as informações apresentadas até aqui, é visível o porquê a maçaranduba é uma das espécies mais exploradas da Amazônia e por isso é dada a relevância de estudar o seu comportamento, para que seja possível a aplicação de manejo adequado para a espécie de forma a garantir a sua conservação na estrutura florestal (CASTRO \& CARVALHO, 2014).

\section{Metodologia}

Área de estudo

Esta pesquisa foi desenvolvida na Floresta Nacional do Tapajós, uma unidade de conservação (UC) localizada no Estado do Pará, ao longo da rodovia Cuiabá - Santarém (BR163), situada entre os paralelos $2^{\circ} 45^{\prime}$ e $4^{\circ} 10^{\prime} \mathrm{S}$ e os meridianos $54^{\circ} 45^{\prime}$ e $55^{\circ} 30^{\prime}$ W. A mesma compreende parte dos municípios de Belterra, Aveiro, Rurópolis e Placas, totalizando uma área de aproximadamente 545.000 hectares (ha).

De acordo com a classificação de Köppen, o clima da região é do tipo Ami (clima tropical chuvoso), com temperatura média de $25,5{ }^{\circ} \mathrm{C}$ anual e maiores precipitações nos meses de janeiro a maio, com média de $1.820 \mathrm{~mm} / \mathrm{ano}$, de forma que região é caracterizada por dois a três meses de seca por ano O tipo de solo predominante na área é o Latossolo Amarelo Distrófico, relevo pouco acidentado com topografia suavemente ondulada. Quanto à vegetação da região, Veloso (1991) a classificou como floresta ombrófila densa, caracterizada por árvores de grande porte. 


\section{Coleta de dados}

Os dados deste trabalho foram coletados na Unidade de Produção Anual (UPA) 12 da Área de Manejo Florestal (AMF) do Anambé, que está inserida dentro da Floresta Nacional do Tapajós, estando esta próxima ao Km 117 da BR 163, rodovia federal que interliga o município de Santarém - PA ao estado de Mato Grosso.

A UPA em questão possui aproximadamente 1.723,66 ha, subdividida em 15 Unidades de Trabalho (UT's) de formato irregular, feitas a partir dos levantamentos gerais do local, resultando em áreas de tamanhos variados entre 74,33 ha e 177,61 ha, como mostra a figura 1. Nestas 15 unidades de trabalho, foi realizado o inventário $100 \%$ de precisão, em que com o auxílio de receptores GPS são coletadas as coordenadas das árvores com diâmetro à altura do peito $(\mathrm{DAP}) \geq 35 \mathrm{~cm}$.

Quanto às equipes de campo, estas foram compostas por um identificador botânico, um anotador, um ajudante (plaqueteiro) e um operador de GPS.

Figura 1. Mapa da localização da (UPA) 12 da unidade de manejo da FLONA do Tapajós.
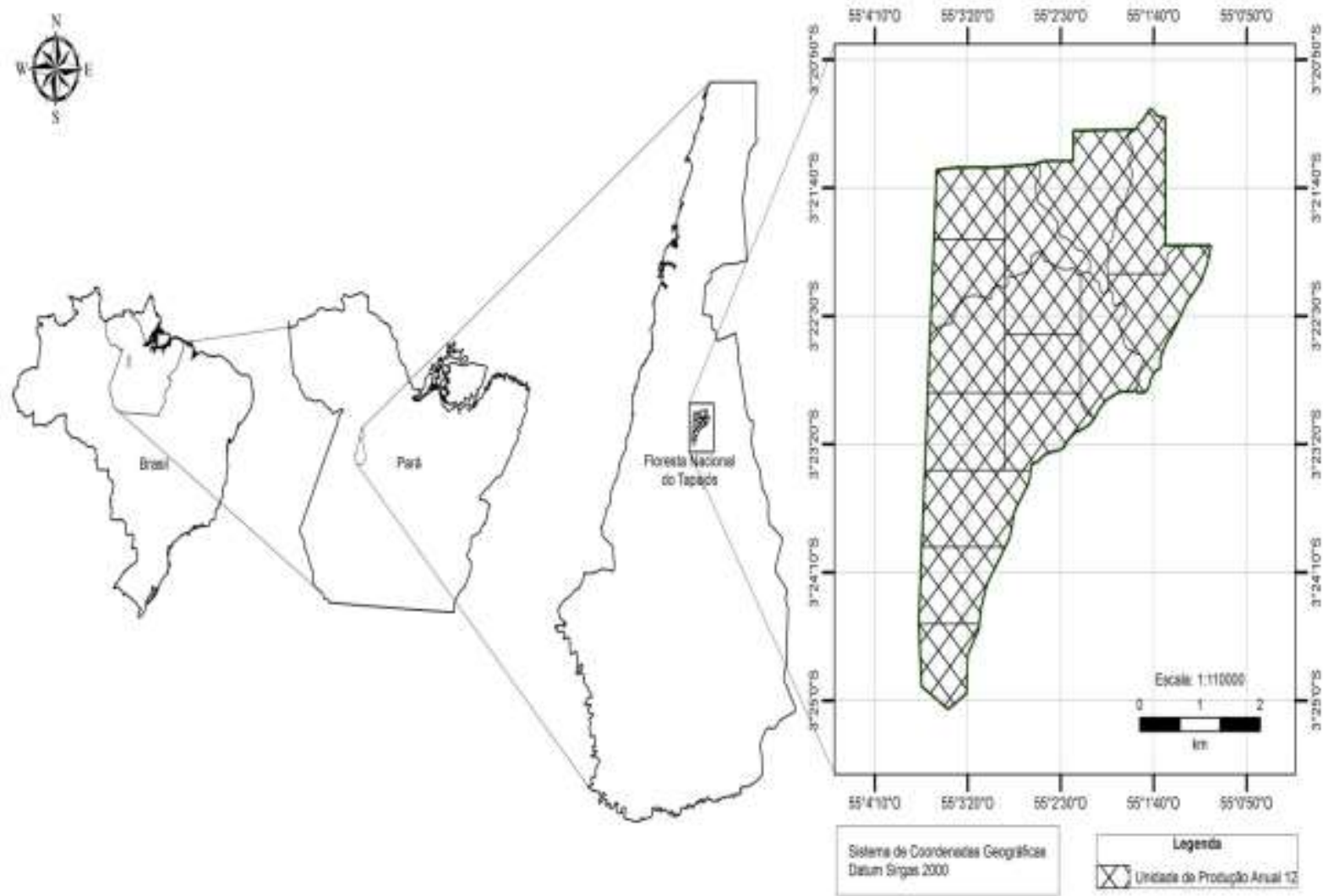


\section{Análise dos dados}

Em relação à estrutura horizontal, conforme recomendado por Müller - Dombois e Ellemberg (1974) foram considerados os seguintes parâmetros para cada espécie: Densidade Absoluta (DA) e Densidade Relativa (DR); Dominância Absoluta (DoA) e Dominância Relativa (DoR); Frequência Absoluta (FA) e Frequência Relativa (FR) e Valor de Importância (VI), conforme demonstrado a seguir.

$$
\begin{gathered}
F A_{i}=100 \frac{u_{i}}{u_{t}} \quad \text { e } \quad \mathrm{FR}_{\mathrm{i}}=100 \frac{F A_{i}}{\sum_{i=1}^{S} F A_{i}} \\
D A_{i}=\frac{n_{i}}{A_{h a}} \text { e } D R_{i}=100 \frac{D A_{i}}{\sum_{i=1}^{S} D A_{i}} \quad D o A_{i}=\frac{G_{i}}{A_{h a}} \text { e } \quad \operatorname{DoR}_{\mathrm{i}}=100 \frac{G_{i}}{\sum_{i=1}^{S} G_{i}}
\end{gathered}
$$

Em que:

$D, D o$, e $F$ correspondem, respectivamente, à densidade, dominância e freqüência, absoluta $(A)$ e relativa $(R)$, da espécie $i$; $n i$ é o número de árvores da espécie $i$; Aha é a área total amostrada em hectares; $S$ é o número total de espécies amostradas; $G i$ é a área basal da espécie $i$ em metros quadrados; ui é o número de parcelas amostrais nas quais a espécie $i$ ocorre; e ut é o número total de parcelas amostrais.

A partir da soma dos valores relativos dessas três estimativas foi calculado o índice do valor de importância (IVI), introduzido por Curtis \& McIntosh (1951).

A estrutura diamétrica foi analisada distribuindo-se as árvores em classes de DAP de $10 \mathrm{~cm}$. Para analisar o incremento periódico entre as medições usou-se intervalo de classe de diâmetro de $10 \mathrm{~cm}$. Consideraram-se todas as árvores vivas observadas na área (Gonçalves e Santos, 2008).

Por sua vez, o índice de Payandeh (1970), foi calculado com base na razão entre a variância do número de árvores por parcela e a média do número de árvores: 


$$
P_{i}=\frac{S_{i}{ }^{2}}{M_{i}} \quad M_{i}=\frac{\sum_{j=1}^{J} n_{i j}}{u T} \quad S_{i}^{2}=\frac{\sum_{j=1}^{J} n_{i j}^{2}-\frac{\left(\sum_{i=1}^{J} n_{i j}\right)^{2}}{u_{T}}}{u_{T}-1}
$$

Em que:

$\mathrm{Si}^{2}=$ variância do número de árvores da i-ésima espécie;

Mi = média do número de árvores da i-ésima espécie;

nij = número de árvores da i-ésima espécie na j-ésima parcela;

$\mathrm{U}_{\mathrm{t}}=$ número total de parcelas amostradas; $\mathrm{e}$

$\mathrm{j}=1,2, \ldots, \mathrm{UT}$.

De acordo com os possíveis resultados obtidos para este índice, considera-se que: se Pi $<1,0$ a espécie apresentou padrão de distribuição espacial do tipo aleatório; se $1 \leq \mathrm{Pi} \leq 1,5$, tendência ao agrupamento e; se $\mathrm{Pi}>1,5$, agrupamento ou agregado.

\section{Resultados e Discussão}

Estrutura horizontal

\section{Abundância}

Observou-se que a maçaranduba foi a espécie mais abundante da UPA 12 da FLONA do Tapajós com 2.995 indivíduos, distribuídos em 1.723,66 ha, representando 6,86\% do total de 100 espécies. Este resultado se assemelha ao resultado encontrado por Barros et al. (2000) em que esta espécie também foi a mais representativa na análise fitossociológica de uma floresta em Curuá - una, estado do Pará, entretanto o valor encontrado por eles foi inferior ao encontrado no presente trabalho, sendo verificados 224 indivíduos em um talhão de 100 ha.

Quanto ao número total de indivíduos do presente estudo, este correspondeu a 1,73 árvores/ha, considerando indivíduos com DAP $\geq 35 \mathrm{~cm}$, enquanto Barros et al. (2000) encontraram o valor de 2,2 árvores/ha, no entanto estes autores consideraram indivíduos com $\mathrm{DAP} \geq 45 \mathrm{~cm}$, além da área de estudo ser bem menor que a deste trabalho, o que pode explicar a divergência de resultados. Em estudo realizado por Costa et al. (1998) em 200 hectares de floresta no município de Moju-PA, foi encontrado o valor de 1,64 árvores/ha, considerando DAP $\geq 45 \mathrm{~cm}$ sendo a maçaranduba a segunda espécie mais abundante na pesquisa. 
É válido salientar que o critério de avaliação dos indivíduos arbóreos com $15 \mathrm{~cm}$ abaixo do DMC permitido por lei $(50 \mathrm{~cm})$, foi adotado pela COOMFLONA para monitorar o estoque de madeira remanescente para o próximo ciclo de corte.

\section{Distribuição Diamétrica}

A distribuição diamétrica da maçaranduba apresentou característica tendendo ao "J invertido", sugerindo desta forma que existe um balanço entre o recrutamento e a mortalidade da espécie, padrão usual das florestas tropicais inequiâneas (BEZERRA et al., 2017). Assim como no trabalho destes autores, é possível observar na figura 2 que as árvores do centro de classe de $45 \mathrm{~cm}$ sofrem uma redução em sua concentração, o que não é esperado para o formato de "J - invertido". Os autores explicam este resultado com o fato do inventário realizado em seu estudo considerar apenas indivíduos comercias passiveis ao corte, conforme a legislação pressupõe.

Já em estudo realizado na AMF da Reserva Extrativista (RESEX) Tapajós - Arapiuns, Costa et al. (2017) verificaram que a espécie Pouteria macrophylla (Lam.) Eyma também apresentou distribuição diamétrica em formato de "J - invertido", onde os mesmos alegaram ser o esperado para florestas nativas, pois revela o potencial que a espécie têm de se recuperar.

Figura 2. Distribuição diamétrica da população de Manilkara huberi (Ducke)A.Chev. da UPA12 da unidade de manejo da FLONA do Tapajós.

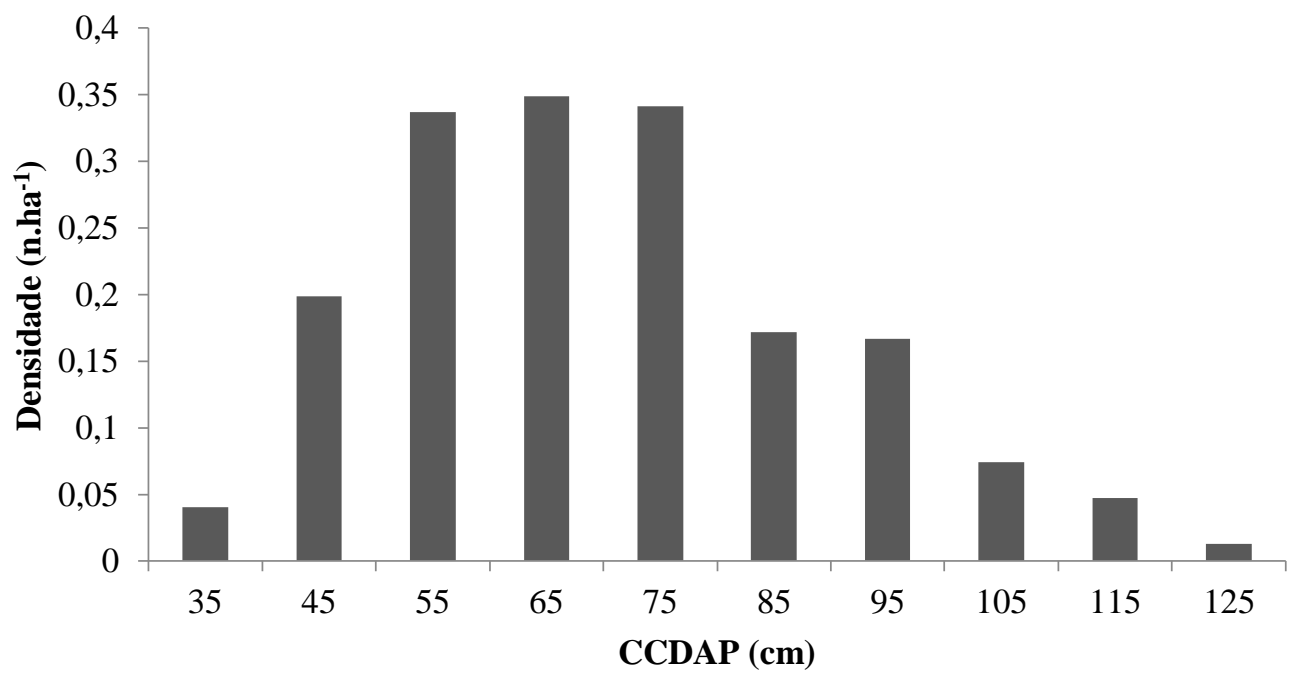


As classes de DAP mais representativas foram as do intervalo entre $55 \mathrm{~cm}-75 \mathrm{~cm}$, resultado semelhante ao encontrado por Hirai et al. (2008) na Fazenda Rio Capim, Paragominas-PA, em que o maior número indivíduos foi encontrado na classe entre $65,0 \mathrm{~cm}$ $74,9 \mathrm{~cm}$.

\section{Volume}

A volumetria da espécie na área estudada foi de $10,46 \mathrm{~m} 3 / \mathrm{ha}$, como mostra a figura 3. Em estudo realizado no município de Moju - PA, Costa et al (1998) descobriram o valor de $8,71 \mathrm{~m}^{3} /$ ha para DAP $\geq 45$ em uma área de 84 ha sendo este valor aproximado ao volume encontrado em nossa pesquisa. Outro trabalho similar foi o de Barros et al. (2000), que encontraram o valor de $13,62 \mathrm{~m}^{3} / \mathrm{ha}$ para DAP $\geq 45$. Sendo assim podemos perceber uma correlação quanto à classe de DAP e volumetria, evidenciando o grande potencial dessa espécie para o manejo florestal nas florestas nativas amazônicas.

Figura 3. Distribuição volumétrica da população de Manilkara huberi (Ducke)A.Chev. da UPA12 da unidade de manejo da FLONA do Tapajós.

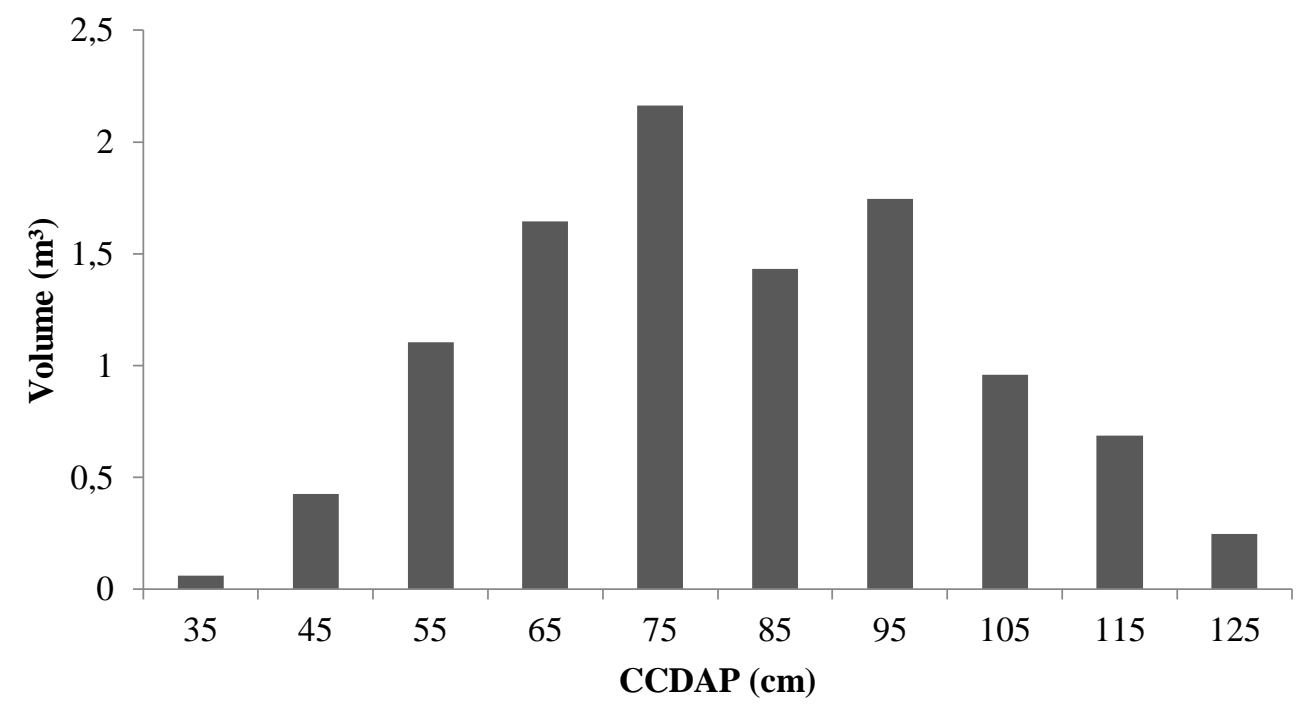

Sabendo disso, Silva \& Ferreira (2016) alertaram que dentre as três espécies florestais com maior volumetria explorada no período de análise de seu estudo, a $M$. huberi já estava inclusa na lista de espécies vulneráveis ou ameaçadas de extinção do Estado do Pará, de acordo com a Resolução do COEMA n54 de 24/10/2007 - Pará. Segundo os autores, este fato alerta para a criação de políticas públicas reguladoras, voltadas para a preservação da 
espécie nos Planos de Manejo Florestal, levando com consideração o grande volume que é explorado anualmente, bem como nos próximos ciclos de corte. Compreende-se, portanto, que é necessário diminuir a pressão sobre as espécies nativas que há séculos vêm sendo exploradas, fomentando pesquisas voltadas para a anatomia e potencialidades de outras espécies.

\section{Distribuição espacial}

O índice de Payandeh $(P)$ usado para avaliar o grau de agregação da espécie pela razão entre a variância do número de maçaranduba por parcela e a média do número de árvores, foi obtido o valor de 45,97 indicando que a espécie ocorre de forma agrupada na Flona do Tapajós. Este fato pode ser possivelmente explicado pela ação de alguns macacos dispersores como o Saimiri sciureus, mas principalmente pelo fato da dispersão da $M$. huberi ocorrer pela gravidade que de acordo com Azevedo et al. (2008) designa uma limitação na eficiência de dispersão de sementes dessa espécie fazendo que as mesmas se mantenham próximas às árvores mãe. Costa et al. (2017) também encontraram o padrão agregado para a $P$. macrophylla, o que é explicado pelos autores como a possibilidade da espécie ter encontrado condições ambientais favoráveis ao seu estabelecimento na área, sobressaindo-se na competição para sua sobrevivência.

Segundo Capretz et al. (2012) o padrão espacial das árvores com processos ecológicos que atuam no ciclo de vida das mesmas revelam como os indivíduos estão organizados horizontalmente no ambiente, sendo a combinação de fatores bióticos e abióticos, podendo ajudar no entendimento da dependência espacial e do padrão de distribuição das espécies. Para Costa et al. (2017) o conhecimento do padrão de distribuição das espécies se faz necessário para a tomada de decisão quanto ao manejo da espécie e escolha dos métodos silviculturais a serem aplicados na área de manejo. Os autores também afirmam que no caso de espécies utilizadas para comercialização madeireira, como a maçaranduba, é viável que se mantenha na floresta alguns indivíduos de tamanho comercial, para que permaneça a ocorrência da sua distribuição natural.

\section{Conclusões}

A Manilkara huberi (Ducke) A. Chev foi a espécie mais presente no local da pesquisa e com grande volumetria encontrada. Desta forma conclui-se que a $M$. huberi demonstra 
grande potencial para o manejo madeireiro de impacto reduzido na Flona do Tapajós e possui boa capacidade de manutenção da biodiversidade, porém torna-se necessário estudos voltados para a adoção de manejo específico para a espécie, tendo em vista que é explorada em grande volumetria anualmente na Amazônia.

\section{Referências}

AZEVEDO, V, R; KANASHIRO, M; GRATTAPAGLIA, D; YAMAGUSHI, C.. Variabilidade no cpDNA em Manilkara huberi, espécie sob manejo sustentável na Amazônia brasileira. Pesquisa Agropecuária Brasileira, v. 43, n. 7, p. 859-867, 2008.

BARROS, A. V. de; BARROS, P. L. C. de; SILVA, L. C. B. da.. Análise fltossociológica de uma floresta situada em Curuá-Uma, Pará. Revista de Ciências Agrarias. Belém, n. 34, p.9$36,2000$.

BEZERRA, R. B. S.; GAMA, J. R. V.; MARTINS, S. V.; MORAES, A.; SANTOS, C. A. A.; CARVALHO, A. N.. Estrutura florestal em projeto de assentamento, comunidade São Mateus, município de Placas, Pará, Brasil. Revista Ceres, Viçosa, v. 60, n.5, p. 610-620, set/out, 2013.

BEZERRA, T. G.; XIMENES, L. C.; CRUZ, G. da S.; GAMA, J. R. V.; SILVA RIBEIRO, R. B.. Estrutura e parâmetros qualitativos de espécies comerciais na comunidade pedreira, Belterra - PA. In: III Seminário de Pesquisa da Floresta Nacional do Tapajós e i Seminário de Pesquisa da Reserva Extrativista Tapajós Arapiuns, Anais, Santarém: UFOPA, 2017.

CAMIZÃO, I.C.; CONTENTE, F.A.S. Distribuição espacial de três espécies arbóreas no fragmento florestal Bosque Rodrigues Alves - Belém, PA. In: Congresso Brasileiro De Sistemas Agroflorestais, 4., 2002, Ilhéus, BA. Anais. Ilheus: Ceplac, 2002. p.1-4.

CAPRETZ RL, BATISTA JLF, SOTOMAYOR JFM, CUNHA CR, NICOLETTI MF, RODRIGUES RR (2012) Padrão espacial de quatro formações florestais do estado de São Paulo, através da Função K de Ripley. Ciência Florestal, 22(3): 551-565.

DOI: 5902/198050986622.

CARVALHO, J. 0. P. de. Distribuição diamétrica de espécies comerciais e potenciais em floresta tropical úmida natural na Amazônia. Belém: Embrapa-CPATU, 1981. 34p (EMBRAPACPATU. Boletim de Pesquisa, 23).

CASTRO, T. C.; CARVAlHO, J. O. P.. Dinâmica da população de Manilkara huberi (Ducke) A. Chev. durante 26 anos após a exploração florestal em uma área de terra firme na Amazônia Brasileira. Ciência Florestal, Santa Maria, v. 24, n. 1, p. 161-169, jan.- mar., 2014.

COSTA, D. H. M.; FERREIRA, C. A. P.; SIllVA, J. N. M.; LOPES, J. do C. A., CARVALHO, J. 0. P. de. Potencial madeireiro de floresta densa no município de Moju, Estado do Pará. Belém: Embrapa - CPATU, 1998. 33p. (Embrapa-CPATU. Documentos, 121). 
COSTA, D. L.; GAMA, J. R. V.; SANTOS, M. F.; RIBEIRO, R. B. S.; MELO, L. O.; FLORES, O. M. M.; SILVA, H. K. M.; CRUZ, G. S.. Estrutura populacional de Pouteria macrophylla (Lam.) Eyma na Reserva Extrativista Tapajós-Arapiuns. Revista Agroecossistemas, v. 9, n. 2, p. 381 - 389, 2017.

CRUZ, E. D.. Germinação de sementes de espécies amazônicas: maçaranduba [Manilkara huberi (Ducke) A. Chev.]. Comunicado Técnico da Embrapa, Março, 2016, Belém, PA

CURTIS, J.T.; MCINTOSH, R.P. 1951. An upland forest continuum in the prairie-forest border region of Wisconsin. Ecology, 32(3): 476-496.

GONÇALVES, F. G.; SANTOS, J. R. Composição florística e estrutura de uma unidade de manejo florestal sustentável na Floresta Nacional do Tapajós, Pará. Acta Amazonica, Manaus, v. 38, n. 2, p. 229 - 244, 2008.

HIRAI, E.H.; CARVALHO, J.O.P.; PINHEIRO, K.A.O. Estrutura da população de maçaranduba (Manilkara huberi Standley) em 84ha de floresta natural na Fazenda Rio Capim, Paragominas, PA. Revista de Ciências Agrárias, Belém, v. 49, p. 65-76, jan./jun. 2008.

LIMA, B. A.; ALMEIDA, B. R. S.; SOUSA, E. A. B.; CRUZ, G. S.; MELO, M. B.; MELO, L. O.; COSTA, D. L.; SANTOS, M. F.. Estrutura e dinâmica florestal sob efeito do manejo madeireiro na FLONA Tapajós. Advances in Forestry Science, Cuiabá, v.5, n.4, p.437-443, 2018.

MUELLER-DOMBOIS, D.; ELLENBERG, G.H. 1974. Aims and methods of vegetation ecology. John Wiley and Sons, Inc., Chichester, England. 547pp.

PARÁ. Resolução COEMA n. 54, de 24/10/2007. Homologa a lista de espécies da flora e da fauna ameaçadas no Estado do Pará. DOE, 2007.

PAYANDEH, B. Comparison of methods for assessing spatial distribution of trees. Forest Science, Bethesda, v. 16, n. 3, p. 312-317, 1970.

SANTANA, A. C.; SANTOS, M. A. S.; SANTANA, A. L.; YARED, J. A. G. O valor econômico da extração manejada de madeira no baixo Amazonas, estado do Pará. Revista Árvore, Viçosa, v.36, n. 3, p.527-536, 2012.

SANTOS, M. G. S.; MELO, L. O.; COSTA, M. S. S.. Efeitos do incêndio florestal sobre a vegetação na Floresta Nacional do Tapajós. Revista Ibero Americana de Ciências Ambientais, v.9, n.7, p.211-223, 2018.

SILVA, J. L.; FERREIRA, T. M. C. Espécies florestais mais exploradas legalmente no estado do Pará no período de 2009 a 2013. Monografia (Graduação em Engenharia Florestal) - Universidade Federal Rural da Amazônia, Paragominas, 2016.

SILVA, K. E.; SOUZA, C. R.; AZEVEDO, C. P.; ROSSI, L. M. B.. Dinâmica florestal, 
estoque de carbono e fitossociologia de uma floresta densa de terra-firme na Amazônia Central. Scientia Forestalis, Piracicaba, v. 43, n. 105, p. 193-201, mar. 2015.

VELOSO, H.P.; RANGEL FILHO, A.L.R.; LIMA, J.C.A. 1991. Classificação da vegetação brasileira, adaptada a um sistema universal. IBGE, Rio de Janeiro, Rio de Janeiro. 123pp.

VIEIRA, D. S.; GAMA, J. R. V.; RIBEIRO, R. B. S.; XIMENES, L. C.. Estrutura, distribuição espacial e volumetria de Carapa guianensis Aubl. na Floresta Nacional do Tapajós. Nature and Conservation, Aquidabã, v.6, n.2, p.18- 25, 2013. 